\title{
Efectos de una intervención educativa frente a la actitud de donar órganos en escolares de la columna de Talcahuano
}

\author{
Jessica Beatriz Constanzo Fuentes ${ }^{1}$, Magali Rodríguez Vidal ${ }^{2}$ \\ ${ }^{1}$ Profesor asistente. Magister en Enfermería Universidad de Concepción. Chile \\ 2 Profesor Asociado. Especialista en Diálisis y Trasplante Renal. Magíster en Enfermería Universidad de Concep- \\ ción, Concepción. Chile
}

\section{Resumen}

Introducción: En Chile el primer trasplante se realizó en el año 1967, desde esa época se ha tratado de motivar a la población para que sea donante. En un comienzo esta medida trajo resultados positivos, pero en los últimos años se ha observado, un retroceso y estancamiento en la actitud pro donación.

Objetivo: Comprobar el efecto de una intervención educativa en la actitud sobre la donación de órganos en escolares entre octavo y segundo medio del establecimiento educacional Santa Bernardita de la comuna de Talcahuano, Concepción (Chile), durante el año 2017.

Material y Método: Estudio cuantitativo, de diseño cuasi experimental (antes-después). La muestra fue no probabilística obtenida por cuotas. Se aplicó el cuestionario que mide actitud ante la donación y preguntas sociodemográficas previo y posterior a la realización de las sesiones educativas.

Resultados: Se contó con una muestra de 136 participantes, con un promedio de edad de 14 años, 75\% profesa una religión, un $42 \%$ pertenece a la clase media baja. Antes de la intervención educativa un 77\% presentó una actitud desfavorable frente a la donación y posterior a ella esta cifra disminuyo a un 59,6\%, siendo estadísticamente significativa.

Correspondencia:

Magali Rodríguez Vidal

Universidad de Concepción.Facultad de Enfermería

Concepción Chile

E-mail: magrodri@udec.cl
Conclusión: Se concluyó que la intervención educativa generó cambios positivos frente a la actitud de donar en esta población, por lo que se sugiere replicar esta metodología en otros establecimientos de la región y a nivel nacional lo que se podría traducir a futuro en un aumento en la cantidad de donantes en Chile.

PALABRAS CLAVE: donación de órganos; actitud; intervención educativa de enfermería.

\section{Effects of an educational intervention in the attitude of donating organs in schoolchildren in the commune of Talcahuano}

\section{Abstract}

Introduction: In Chile, the first transplant was performed in 1967, since then, many efforts have been made to motivate the population to become a donor. At first, this measure brought positive results, but in recent years it has been observed a regression and stagnation in the pro-donation attitude.

Objective: To verify the effect of an educational intervention on the attitude about organ donation in students between $8^{\text {th }}$ grade primary school and $2^{\text {nd }}$ grade secondary school from the 'Fundacion Educacional Santa Bernardita' school of Talcahuano, during the year 2017.

Material and Method: Quantitative study, of quasiexperimental design (before-after). The sample was nonprobabilistic obtained by quotas. The questionnaire that measures attitude to donation and sociodemographic 
questions before and after the educational sessions were used.

Results: The sample consisted on 136 participants, with an average age of 14 years, $75 \%$ profess a religion, $42 \%$ belong to medium-low class. Before the educative intervention, $77 \%$ showed a negative attitude towards donation and after the intervention, the number decreased to $59.6 \%$, being statistically significant.

Conclusions: It was concluded that the educational intervention generated positive changes in response to the attitude of donating in this population, so it is suggested to replicate this methodology in other educational centers in the region and at the national level, which could generate an increase in the number of donors in Chile.

KEYWORDS: organ donation; attitude; education in nursing.

\section{Introducción}

A nivel mundial existe una enorme preocupación respecto a la donación de órganos para trasplante, ya que cada año va en aumento la brecha que existe entre la oferta y la demanda, esta situación genera un aumento en la mortalidad de la población ya que muchos de los pacientes que están en las listas de espera fallecen al no poder obtener un órgano a tiempo ${ }^{1}$.

Dentro de los países pioneros en el proceso de donación de órganos destaca España, que tras la creación de la Organización Nacional de Trasplante ha avanzado de 14 donantes por millón de población (pmp) a 36 donantes pmp en el año 2014, este progreso tan importante ha sido por la aplicación del Modelo Español, que aplica medidas dirigidas a la mejora en el proceso de la donación, sugerido por la Organización Mundial de la salud ${ }^{2,3}$.

Una de las características principales que presenta este modelo, es que el equipo coordinador es multidisciplinario y organizado a nivel nacional, (médicos intensivistas y enfermeras/os) formados en la promoción, educación permanente y continua, tanto a nivel comunitario, escolar y familiar ${ }^{4}$. En Chile, la realidad se aleja bastante de las cifras presentadas en España, y a nivel internacional. A pesar que se modificó la normativa existente en el año 2013 por la ley 20.413 sobre donación de órganos la cual establece nuevas normas, siendo una de las más relevantes la determinación de que todos los ciudadanos son donantes a menos que la persona manifieste lo contrario de forma expresa, en el Servicio de Registro Civil. En esta ley, se destacan también dentro de sus objetivos, realizar actividades de difusión, educación y sensibilización de la opinión pública, crear y fortalecer grupos de apoyo y contención emocional a la familia,

Según el Instituto de Salud Pública de Chile en su registro nacional de trasplante durante el año 2014, el número de pacientes en lista de espera de donante cadáver correspondió, a 1794 (riñón: 1585, páncreasriñón 8; hígado: 125, corazón: 16, pulmón 60), cifras que van en aumento, otro dato significativo es que en el año 2016 solo se registraron 7,9 donantes efectivos por millón de habitantes, cifras alejadas de la necesidad nacional. A lo expuesto se suma que en el año 2016 se registró un $51 \%$ de negativa ante la donación a nivel nacional ${ }^{7}$.

De acuerdo a lo anterior, se han identificado en la literatura tanto a nivel nacional como internacional, múltiples factores que influyen en una actitud desfavorable frente a la donación, destacando: miedo que presentan ante la atención medica que ellos recibirían en una situación de urgencia, desconocimiento de cómo se manipularía el cadáver de su familiar o si este sentirá dolor al momento de donar, religiosidad y factores socioculturales de décadas derivadas de la relación con la propiedad del cuerpo humano, tráfico de órganos y confusión de conceptos muerte encefálica y estado de coma. Todos los aspectos mencionados están unidos por un factor común: el desconocimiento en relación a estos temas. En la bibliografía no se encontraron apenas investigaciones nacionales e internacionales que estuvieran enfocadas a la intervención educativa, tanto a nivel escolar como comunitario, sobre esta problemática ${ }^{4,7}$, hecho que debería tenerse en cuenta, teniendo en cuenta que las listas de espera para trasplante de órganos irán en aumento, por la prevalencia de las enfermedades crónicas no transmisibles determinadas como una pandemia a nivel mundial ${ }^{8}$.

El concepto donación es definido por la Real Academia de la Lengua como la "liberalidad de alguien que transmite gratuitamente algo que le pertenece, a favor de otra persona que lo acepta" ${ }^{9 \prime}$. Además debemos considerar que esta decisión se caracteriza como "un gesto voluntario y de gran bondad hacia los demás" y "tiene sólo un fin terapéutico, sin recibir ningún tipo de compensación ${ }^{3,10}$. 
En este sentido, enfermería como disciplina y profesión gestora del cuidado, tiene la obligación de generar intervenciones en diferentes áreas sociales, siendo la promoción en salud un pilar importante a utilizar, a través de la educación, como herramienta de bajo costo, alto impacto y trascendencia en la salud de la comunidad.

Por todo ello, nos planteamos la siguiente pregunta de investigación: ¿Cuál es el efecto que genera una intervención educativa en la actitud hacia la donación de órganos?, con el objetivo general de comprobar la eficacia de una intervención educativa en la actitud hacia la donación de órganos en escolares que cursan la enseñanza media, en Chile.

\section{Material y Método}

Ámbito: Establecimiento educacional de la Comuna de Talcahuano, Concepción, Chile. El estudio se llevó a cabo durante el primer semestre del año 2017 a escolares entre los 14 a 16 años.

Diseño: Se ha llevado a cabo un estudio cuasi experimental, prospectivo (Pre-test Pos-test), en el mismo grupo de alumnos.

Población y muestra: Se tomó muestra de tipo no probabilística por cuotas, inicialmente formada por 162 estudiantes que cursaban la enseñanza media, según los registros del establecimiento. La muestra final se conformó por 136 participantes que cumplieron con los criterios de inclusión establecidos a continuación.

\section{Criterios de inclusión}

- Ser alumno regular del establecimiento educacional "Colegio Santa Bernardita".

- Debe estar cursando enseñanza media al momento de la realización de la intervención educativa.

- Que el apoderado del menor firme el consentimiento informado para la participación de su pupilo en la investigación.

- Que es escolar firme el asentimiento informado para la participación de la investigación.

Recogida de datos: El procedimiento de recolección de datos, se realizó a través de 5 visitas, previa organiza- ción con los directivos académicos del establecimiento, donde se aplicó el instrumento en dos oportunidades y se realizaron 3 sesiones educativas. Todos los participantes fueron informados acerca de la investigación y firmaron voluntariamente el Consentimiento y asentimiento Informado.

Instrumento de medida: Para evaluar la actitud se utilizó el instrumento recolector de datos denominado "Actitud frente a la donación de órganos en escolares" auto aplicado por los escolares, y constó de 2 secciones. Primera sección: con preguntas sociodemográficas (edad, sexo, ingreso económico, religión, número de personas del núcleo familiar, comunicación familiar sobre dedición de donar) y segunda sección: cuestionario para medir la actitud hacia la donación, de Fernández, Zapata, Díaz y Taypicahuana (Perú, 2014), constituido por 24 preguntas distribuidas en 4 items, dimensiones: económica (5), social (7), ético-moral (7) y cultural (5), el cual fue validado a nivel de constructo a través de la aplicación del test de adecuación de Kaiser-Meyer- 0Ikin con un resultado de 0,696 y esfericidad de Barlet con un valor menor a 0,005, y para la evaluación de los ítems, se usó el Alfa de Cronbach, cuyo resultado fue superior a 0,7 en todos ellos ${ }^{11}$. Las 24 preguntas se estructuraron en formato Likert con 5 opciones de respuesta (totalmente desacuerdo, desacuerdo, indeciso, acuerdo, totalmente acuerdo) con un total de 120 puntos, generándose dos categorías, una sumatoria menor o igual a 82 puntos: corresponde a una actitud desfavorable ante la donación de órganos, y un puntaje superior a los 82 puntos: una actitud favorable.

Aspectos éticos: El estudio conto con la aprobación del comité de Ética de la Facultad de Enfermería. Se aplicó el consentimiento informado y firmado a los padres/ tutores y asentimiento informado y firmado a los estudiantes, considerando los 7 requisitos éticos planteados por Ezekiel Emanuel.

Análisis estadístico: Los datos obtenidos se vaciaron a una planilla Excel V2010, y analizados en los softwares R-Project, SPSS V. 19.00. Se consideró un nivel de significación para $p<0,05$. Para la estadística descriptiva se empleó distribución de frecuencias, medidas de tendencia central y medidas de dispersión (rangos y desviación estándar). Para el análisis inferencial se utilizó el Test no Paramétrico de Wilcoxon, prueba Chi-Cuadrado de independencia y prueba de Mcnemar para comparación de proporciones de muestras pareadas o dependientes. 


\section{Resultados}

En la Tabla 1 están representadas las variables sociodemográficas, como la edad, sexo, creencias religiosas, practicar religión, ingresos económicos y número de personas que viven en el hogar.

Tabla 1. Resultado variables independientes.

\begin{tabular}{|c|c|c|}
\hline \multicolumn{3}{|l|}{ Variables independientes } \\
\hline Edad & Valor & \\
\hline Mínimo & 13 & \\
\hline Mediana & 14 & \\
\hline Máximo & 16 & \\
\hline Sexo & $\mathbf{N}$ & $\%$ \\
\hline Masculino & 64 & $47,1 \%$ \\
\hline Femenino & 72 & $52,9 \%$ \\
\hline Creencias religiosas & $\mathbf{N}$ & $\%$ \\
\hline Sí & 102 & $75,0 \%$ \\
\hline No & 34 & $25,0 \%$ \\
\hline Religión que practican $(n=102)$ & $\mathbf{N}$ & $\%$ \\
\hline Católica & 65 & $63,7 \%$ \\
\hline Evangélica & 32 & $31,4 \%$ \\
\hline Testigo de Jehová & 3 & $2,9 \%$ \\
\hline Mormón & 2 & $2,0 \%$ \\
\hline Otra & 0 & $0,0 \%$ \\
\hline Promedio de Ingreso Económico (\$) & $\mathbf{N}$ & $\%$ \\
\hline 0 a 250.000 & 6 & $4,4 \%$ \\
\hline 250.000 a 400.000 & 27 & $19,9 \%$ \\
\hline 400.000 a 900.000 & 58 & $42,6 \%$ \\
\hline 900.000 a 1.200 .000 & 35 & $25,7 \%$ \\
\hline Más de 1.200 .000 & 10 & $7,4 \%$ \\
\hline $\mathbf{N}^{\circ}$ Personas que viven en el hogar & Valor & \\
\hline Mínimo & 2 & \\
\hline Mediana & 4 & \\
\hline Máximo & 13 & \\
\hline Promedio & 4,33 & \\
\hline
\end{tabular}

Fuente: "Actitud frente a la donación de órganos en escolares de la comuna de Talcahuano 2017".

La Tabla 2 muestra cómo se incrementó el porcentaje de alumnos que habían tenido una conversación familiar sobre la donación de órganos, después de la intervención educativa.

En la Tabla 3 puede observarse la modificación de la actitud frente a la donación de órganos, tras la intervención educativa.
Tabla 2. Comunicación en la familia sobre la decisión de donar pre y post intervención.

\begin{tabular}{|c|c|c|c|c|}
\hline & \multicolumn{2}{|c|}{$\begin{array}{l}\text { Pre } \\
\text { intervención }\end{array}$} & \multicolumn{2}{|c|}{$\begin{array}{l}\text { Post } \\
\text { Intervención }\end{array}$} \\
\hline $\begin{array}{l}\text { Comunicación far } \\
\text { sobre la decisión } \\
\text { de donar }\end{array}$ & $\mathbf{N}$ & $\%$ & $\mathbf{N}$ & $\%$ \\
\hline Sí & 63 & $44,7 \%$ & 82 & $60,3 \%$ \\
\hline No & 78 & $55,3 \%$ & 54 & $39,7 \%$ \\
\hline
\end{tabular}

Fuente: "Actitud frente a la donación de órganos en escolares de la comuna de Talcahuano 2017".

Tabla 3. Actitud frente a la donación de órganos pre y post intervención.

\begin{tabular}{|c|c|c|c|}
\hline & $\begin{array}{r}\text { Acti } \\
a t \\
\text { (Post) } \\
\end{array}$ & $\begin{array}{l}\text { d frente } \\
\text { lación } \\
\text { tervención }\end{array}$ & \\
\hline $\begin{array}{l}\text { Actitud frente } \\
\text { a donación (Pre) } \\
\text { Intervención }\end{array}$ & Favorable & Desfavorable & Totales \\
\hline Favorable & $22,1 \%$ & $0,0 \%$ & $22,1 \%$ \\
\hline Desfavorable & $37,5 \%$ & $40,4 \%$ & $77,9 \%$ \\
\hline Totales & $59,6 \%$ & $40,4 \%$ & \\
\hline
\end{tabular}

Fuente: "Actitud frente a la donación de órganos en escolares de la comuna de Talcahuano 2017".

Para medir la efectividad de la intervención educativa se utilizó la prueba de Mcnemar, cuyo resultado reportó que hubo diferencias estadísticamente significativas pre y post educación $\left(\chi^{2}=49,02, g \mid=1, p=0,000\right)$.

En la Tabla 4 se representan los resultados de la prueba chi-cuadrado. Estos indican que la única variable sociodemográfica que se relacionó con la actitud frente a la donación, fue la Religión que practican o de la que se sienten parte los estudiantes $(p=0,02)$.

Tabla 4. Relación de la actitud frente a la donación de órganos y las variables sociodemográficas cualitativas.

\begin{tabular}{lcc}
\hline & Estadístico & Valor-P \\
\hline Sexo & 0,33 & 0,57 \\
\hline Religión & 5,7 & $0,02\left(^{*}\right)$ \\
\hline Ingreso Económico & 1,52 & 0,82 \\
\hline Comunicación Familiar & 0,44 & 0,51 \\
\hline \hline
\end{tabular}

Fuente: "Actitud frente a la donación de órganos en escolares de la comuna de Talcahuano 2017". 
Finalmente, se realizaron pruebas para relacionar con variables cuantitativas, pero no se encontró ninguna relación estadísticamente significativa.

\section{Discusión}

La muestra tuvo un promedio de 14 años edad lo que se asemeja a lo planteado por los referentes empíricos, que nos señalan que a menor edad hay una mayor disposición a la recepción y trasmisión de información, llevándolos a ser importantes agentes de cambio en la población, mencionando a su vez que los padres y/o apoderados de los menores, sugieren iniciar la educación sobre la donación entre estos rangos de edad ${ }^{12,13}$.

Respecto al ingreso económico se evidencia que un mayor porcentaje de los encuestados se encuentra en la clase media baja, con un ingreso promedio de quinientos mil pesos (676 euros) por grupo familiar. La literatura menciona que a menor ingreso económico, existiría una tendencia desfavorable frente a la actitud de donación, estableciendo que esta población tendría una menor posibilidad de acceder a educación de calidad lo que generaría mayor desconfianza en el proceso de procuramiento, reflejándose en una baja adhesión a ser donante ${ }^{13,14}$. En la presente investigación, esta variable no presentó una relación estadísticamente significativa, similar a lo reportado por Neira et al. Lo que lleva a plantearse que para la población chilena, este factor no sería una limitante al considerar futuras planificaciones educativas en este grupo etario ${ }^{14}$.

La religión en este estudio, fue la única variable que presentó una relación estadísticamente significativa frente a la actitud de donación; se observó que el $75 \%$ de los adolescentes profesaban una religión, principalmente católica y evangélica. La creencia popular tiende a relacionar en forma negativa la religión y la actitud de donación, estableciendo que ciertas religiones la sancionan, pero el catolicismo ve a la donación como un "acto de caridad y de amor hacia el prójimo", los testigos de Jehová tienen la visión de que "cada persona debe tomar su propia decisión y mencionan que si los órganos vienen limpios de sangre antes de trasplantar no sería ningún problema el realizar este procedimiento". Dado lo anterior se observa una dicotomía entre lo establecido como norma de una religión y la actitud de las personas frente a la donación, lo cual, reafirma Castañeda et al. donde un $20 \%$ de la población encuestada se niega a la donación de órganos poniendo a la religión como causa de su postura, ante esto surge la necesidad de profundi- zar cualitativamente, sobre el sentir de los usuarios frente a este tema que puede estar influyendo de manera significativa en la cantidad de donaciones efectivas existentes en nuestro país, hallazgo importante a considerar en intervenciones educativas a futuro, trasmitiendo a la población que las grandes religiones llaman a sus fieles a ser donantes ${ }^{13-15}$.

La comunicación en vida de la decisión de querer ser donante fue un factor predictor de una donación efectiva, importante a considerar en nuestra realidad nacional ya que serán los familiares quienes tomarán la decisión final de realizar o no la donación. Diversas investigaciones, mencionan que un bajo nivel de comunicación familiar está asociado a una actitud indecisa o negativa, lo que conlleva a que se generen menos donantes efectivos ${ }^{16,12-14}$. En la presente investigación más de la mitad de la muestra refirió no haber conversado esta temática en familia, lo que se constató, por una actitud desfavorable en más de la mitad de la población antes de la intervención educativa, situación que cambió favorablemente después de la realización de la misma, lo que nos demuestra la relevancia de actuar a temprana edad y lograr que este tema sea tratado de manera natural y permanente en la familia y no solo cuando hay campañas televisivas o algún caso mediático en los medios de comunicación.

En relación a la actitud que presentaron los escolares frente a la donación, se pudo establecer que antes de la intervención educativa hubo una respuesta mayoritariamente desfavorable $(77,9 \%)$, la que se modificó significativamente $(40,4 \%)$, posterior a ella, similar a lo reportado por otros autores ${ }^{17,18}$. En este sentido, la bibliografía advierte que la actitud frente a la donación es una problemática que se debe abarcar considerando diversas aristas y evaluando el contexto sociocultural de la población en un momento determinado. El principal detractor de una actitud desfavorable ante la donación, es la falta de conocimientos, generando mitos, ideas erróneas, desconfianza y ansiedad ${ }^{19}$.

La realización de contenidos educativos para promover la donación de órganos, desde el nivel escolar no solo busca aumentar las donaciones efectivas, sino de manera paralela, empoderar a la comunidad para que estos tomen una decisión basada en evidencia científica, actualizada y fidedigna, los cuales deben ser adecuados a cada edad y contexto sociocultural, y así generar cambios paulatinos y permanentes en el entorno social ${ }^{20}$. Para lograr este objetivo de traspaso de información, los profesionales de enfermería cumplen 
un rol relevante dentro del equipo de salud, quienes se desempeñan en diversas áreas, desarrollando funciones en el ámbito asistencial, gestión, investigación y educación en salud constituyéndose este último, en uno de los eslabones más importante en la trasmisión de información, específicamente en la atención primaria donde su objetivo principal es la promoción y prevención en salud. Dentro de las medidas de acción que desarrollan, en la actualidad están las intervenciones educativas a nivel escolar, pero no se considera la temática de donación y trasplante por no estar incorporada en los planes curriculares del Ministerio de Educación y tampoco figura entre los temas relacionados con la educación en salud para la comunidad.

En esta investigación los estudiantes consideraron altamente positivo que el profesional enfermero/a fuese el encargado de entregar estos contenidos relacionados a la donación, dada la cercanía permanente con la comunidad abarcando su quehacer en todas las edades del ciclo vital. Dado lo anterior surge la interrogante ¿Por qué esperar a que algo catastrófico ocurra a nivel personal o mediático, para comenzar a hablar de la donación de órganos? ${ }^{21,22}$. Al vista de estos resultados y avalados por las referencias bibliográficas, se ve la necesidad de instaurar esta temática como contenido obligatorio dentro de las políticas públicas considerando el binomio salud educación, generando intervenciones multidisciplinarias en todos los niveles de atención, logrando trascendencia y continuidad en la transmisión de información. Lo anterior, significaría una modificación del modelo imperante, en el cual la mayor acción que realizan los profesionales de la salud involucrados en este proceso de donación, se lleva a cabo en el ámbito intrahospitalario principalmente al momento de pesquisar a un potencial donante, que como se ha demostrado ampliamente en la literatura, no es el momento más adecuado para realizar la solicitud de donación si la comunidad no ha estado previamente sensibilizada con el tema. La principal demanda que presentan los familiares de los potenciales donantes es información y esta la requieren directamente del equipo de salud ya que ellos les brindan confianza y seguridad durante todo el proceso $0^{15,21,22}$.

En este estudio se pudo comprobar que intervenciones educativas en la etapa escolar podrían generar un cambio positivo frente a la actitud de donación, lo que podría comportar a largo plazo, un aumento en el número de donaciones efectivas, con todos los beneficios que ello conlleva en las personas que dependen de la donación de un órgano para mejorar su calidad de vida y disminución de los costos en salud del paciente con enfermedad renal crónica terminal y en tratamiento de sustitución renal.

A la vista de estos resultados podemos concluir que una intervención educativa en escolares, sobre la actitud de donar órganos, fue eficaz en la modificación de la actitud de los mismos, altamente positiva, de bajo costo económico y que ayudo a instaurar esta temática dentro del ámbito familiar.

Recibido: 17 agosto 2018

Revisado: 20 agosto 2018

Modificado: 12 septiembre 2018

Aceptado: 20 septiembre 2018

\section{Bibliografía}

1. Escudero D, Otero J. Intensive care medicine and organ donation: Exploring the last frontiers? Med intensiva, 2015; [Consultado 2 agosto 2018];39(6): 373-81. Disponible en: https://www.ncbi.nlm.nih. gov/pubmed/25841298

2. Ministerio de sanidad, servicios sociales e igualdad. España vuelve a batir su record de donación y trasplante, con 36 donantes p.m.p y un total de 4360 pacientes trasplantados. Gabinete de prensa Madrid [Internet], 2014; [Consultado 1 agosto 2018];1-6. Disponible en: http://www.ont.es/prensa/NotasDePrensa/13\%20Ene\%2015\%20NP\%20Balance\%20Donacion\%20y\%20Tx\%202014.pdf

3. Organización nacional de trasplante, gobierno de España [Internet]. España; [Consultado 5 junio 2018]. Disponible en: http://www.ont.es/publicaciones/Paginas/default.aspx

4. Knihs NS, Schirmer J, Roza BA. Adaptación del modelo español de gestión en trasplante para la mejora en la negativa familiar y mantenimiento del donante potencial. Texto Contexto enferm, Florianapolis [Intenernet], 2011; [Consultado 5 mayo 2018];20 (6):59-65. Disponible en: http://www.scielo.br/scielo.php?pid=S0104-07072011000500007\&scrip$\mathrm{t}=\mathrm{sci} \_$abstract\&tlng=es 
5. Bustamante-Ubilla M, Villarreal-Navarrete P. Motivación a la donación de órganos en Chile. Panorama Socio económico [Internet], 2008 [Consultado 8 junio 2018];26(36):86-97. Disponible en: http:// www.redalyc.org/articulo.oa?id=39903608

6. Corporación del trasplante [Internet]. 2015 Mayo. [Consultado 8 junio 2018] Disponible en: http:// www.sociedaddetrasplante.cl/

7. International Registry in Organ Donation and Trasplantation (2012). Second report data. [Internet]. 2011. [Consultado 8 marzo 2018]. Disponible en: http://www.trasplante.cl/noticias/internacionales/205-20-reporte-internacional-donacion-ytrasplantes-de-organos.html

8. Enfoque de riesgo para la prevención de enfermedades cardiovascular. Minsal, 2014 [Consultado 10 mayo 2018]. Disponible en: http://www.enfermeriaaps.com/portal/download/ENFERMEDADES\%20CARDIOVASCULARES/Enfoque $\% 20$ de\%20riesgo\%20para\%20la\%20prevencion\%20 de $\% 20$ enfermedades $\% 20$ cardiovasculares. $\% 20$ MINSAL\%20Chile\%202014.pdf

9. Real-Academia-de-la-lengua-Española. Real Academia Española. [0nline]. Búsqueda de Términos: "Donación". 2015. [Consultado 25 marzo 2018] Disponible en: http://lema.rae.es/drae/srv/search?i$\mathrm{d}=0$ EKtAnnguDXX27sn7w3S

10. Mercado - Martínez Francisco, Padilla- Altamirano César, Díaz Medina Blanca, Sánchez - Pimienta Carlos. La visión del personal de salud sobre la donación y el trasplante de órganos: una revisión de la literatura. Texto Contexto Enferm, Florianapolis [internet]. 2015 [Consultado 27 junio 2018]; 24(2):574-3. Disponible en: http://www.scielo.br/ pdf/tce/v24n2/es_0104-0707-tce-24-02-00574. pdf

11. Fernández-Rioja F, Zapata-Zapata C, Díaz-Vélez C, Taypicahuana-Juárez J. Validacion de instrumento para medir la actitud hacia la donanion de órganos en familiares de pacientes hospitalizados. Rev Cuerpo Med. [Intnernet] 2014 [Consultado 25 marzo 2018];7(1);24-8. Disponible en: file:///C:/Users/Patricia/Downloads/Dialnet-ValidacionDeInstrumentoParaMedirLaActitudHaciaLaDo-4755890\%20(3). pdf
12. Siebelink M, Albers $M$, Roodbol $P$, Van de Wiel H. Estudio exploratorio en línea sobre las opiniones de los padres respecto de la donación de órganos: de las decisiones individuales a las conversaciones en familia. Arch Argen Pediatr. 2014;112 (3):224-30.

13. Castañeda-Millán DA, Alarcón $F$, Ovalle $D$, Martínez $C$, Marcela-González L, Burbano-Perea $L$, et al. Actitudes y creencias sobre la donación de órganos en Colomba: ¿Dónde se deben enfocar los esfuerzos para mejorar las tasas naciones de donación? Rev. Fac. Med. [Intnernet] 2014 [Consultado 15 junio 2018];62(1):17-5. Disponible en:https:// revistas.unal.edu.co/index.php/revfacmed/article/ view/43660

14. San Martin H, Navarro Saldaña G, Jofré Oliden I. Factores asociados a la disposición hacia la donación de órganos en una muestra chilena. Rev. Estudiantes Esc. De psicología, Univ de Costa Rica. 2017;12 (1):69-74.

15. Vélez E. Donación de órganos, una perspectiva antropológica. Rev Soc Esp Enferm Nefrol 2007;10 (3):179-5.

16. Doggenweiler I, Guic E. Determinantes psicosociales de la intención de donación de órganos en una muestra chilena. Rev. méd. Chile. 2014;142(1):27-3.

17. Aguilar-Fernández AM, Correa-López LE, De La Cruz-Vargas JA. Actitudes de estudiantes universitarios acerca de la donación de órganos en población latinoamericana. [Artículo de Revisión]. Rev. Fac. Med. Hum. 2017;17(2):81-9. Disponible en: http://v-beta.urp.edu.pe/pdf/id/9165/n/actitudes-de-estudiantes-universitarios-acerca-de-la-donacion-de-organos-en-poblacion-latinoam

18. Villalón Santillán S. Actitudes de jóvenes universitarios acerca de donación de órganos y tejidos. Latinoamericana: experiencias, desafíos y compromisos sociales. México: Asociación Mexicana de Alternativas en Psicología [Intnernet]; 2014 [Consultado 25 mayo 2018]; p. 399-400. Disponible: https://www.researchgate.net/profile/Viridiana_Pelaez_Hernandez/publication/268818971_Psicologia_Latinoamericana_Experiencias_desafios_y_ compromisos_sociales_PARTE_III_FORMACION INTERVENCION E INVESTIGACION EN_EL_PROCESO_SALUD-ENFERMEDAD/ links/547902050cf205d1687f7cbf/Psicologia-La- 
tinoamericana-Experiencias-desafios-y-compromisos-sociales-PARTE-III-FORMACION-INTERVENCION-E-INVESTIGACION-EN-EL-PROCESO-SALUD-ENFERMEDAD.pdf

19. Mora T, Rodríguez F, Salcido P, González M, Martínez M, Sánchez P. Encuesta de opinión sobre donación de órganos 2009. Med Int Mex [Intnernet] 2010 [Consultado 15 julio 2018];26:313-8. Disponible en: http://www.medigraphic.com/pdfs/medintmex/mim-2010/mim104c.pdf

20. Luque Vadillo E, Matamala A, Torres Quintana A, Barroso Ortín R, Sánchez Villalba Y, Ferret Piñera $A$ et al. Evaluación de un programa educativo sobre la donación de órganos dirigido a escolares. Rev Soc Esp Enferm Nefrol. 2006;9(3):25-9.
21. Conesa Bernal C, Rios Zambudio A, Ramírez Romero $\mathrm{P}$, Rodríguez Martínez MM, Canteras Jordana M, Parrilla Paricio P. Importancia de los profesionales de atención primaria en la educación sanitaria de la donación de órganos. Aten Primaria [Intnernet]. 2004 [Consultado 11 julio 2018]:34 (10):528-5. Disponible en: http://www.elsevier.es/ es-revista-atencion-primaria-27-articulo-importancia-los-profesionales-atencion-primaria-13069582

22. M. Strauck Franco, C. Guillen Arruda. Twitter como herramienta para comunicación en salud en el contexto del fomento de la donación de órganos en el Perú. Zer. [Intnernet] 2017 [Consultado 5 junio 2018]:22(42):189-09. Disponible en: http://www. ehu.eus/ojs/index.php/Zer/article/view/17841

Este artículo se distribuye bajo una Licencia Creative Commons Atribución-NoComercial 4.0 Internacional. https://creativecommons.org/licenses/by-nc/4.0/

Open Access (c) () () 